\title{
Xinmailong mitigated epirubicin-induced cardiotoxicity via inhibiting autophagy
}

Hui $\mathrm{Li}^{1}$, Yiqing Mao ${ }^{1}$, Qun Zhang ${ }^{1}$, Qing $\operatorname{Han}^{1}$, Zhenming Man ${ }^{1}$, Jingyu Zhang ${ }^{1}, \mathrm{Xi}_{\text {Wang }}{ }^{1}$, Ruobi

$\mathrm{Hu}^{1}$, Xuehui Zhang ${ }^{1}$, David M Irwin ${ }^{3^{*}}$, Gang Niu ${ }^{2 *}$, Huanran Tan ${ }^{1 *}$

1. Department of Pharmacology, Peking University, Health Science Center, Beijing 100191, China;

2. Beijing N\&N Genetech Company, Beijing, China;

3. Department of Laboratory Medicine and Pathobiology, University of Toronto, Toronto, Ontario M5S1A8, Canada;

*These authors contributed equally to this work.

To whom correspondence should be addressed:

Huanran Tan, Department of Pharmacology, School of basic medical science, Peking University, 38

XueYuan Road, Beijing 100191, China, Tel: +861082802004, Fax: +861082802004; E-mail:

tanlab@bjmu.edu.cn

Gang Niu, Beijing N \& N Genetech Company, Ltd. Beijing 100082, China, Tel:+861064047343

Fax:+861064031254; E-mail: nngene@sohu.com

David M Irwin, Department of Laboratory Medicine and Pathobiology, University of Toronto,

Toronto, Ontario M5S1A8, Canada, Tel:+14169780519, Fax:+14169785959; E-mail:

david.irwin@utoronto.ca 


\begin{abstract}
Ethnopharmacological relevance:

Using insects, such as the cockroach, for the treatment of disease has a long history in traditional Chinese medicine. Xinmailong (XML) Injection, a bioactive composite extracted from Periplaneta americana (a species of cockroach), shows reasonable protective effects against cardiovascular injury and was approved for the use in the treatment of cardiac dysfunction in 2006, yet its cardio protective mechanisms remain unclear.
\end{abstract}

Aim: The present study aims to examine the protective effects of XML against epirubicin-induced cardiotoxicity in vivo and determine its underlying mechanisms.

\title{
Materials and Methods:
}

The chemical characteristics of XML were identified using high performance liquid chromatography (HPLC). Rats were intraperitoneally injected with epirubicin and then treated with XML for 14 days. Survival rate, echocardiography, electrocardiographic recordings and Masson staining were used to evaluate the cardioprotective activity of XML. Western blot and quantitative real time reverse transcriptase polymerase chain reaction (RT-PCR) analyses were used to investigate the molecular mechanisms underlying the actions of XML.

\section{Results:}

XML treatment significantly enhanced the survival rate of rats from epirubicin-induced heart failure. XML prevented left ventricle dilatation, improved cardiac function. Furthermore, treatment with XML also significantly inhibited the accumulation of collagen, reduced the levels of mRNA for matrix metalloproteinases-9 (Mmp9) and transforming growth factor- $\beta$ 1(Tgfb1). This action of XML therefore might be responsible, at least in part, for the attenuation of cardiac fibrotic remodeling. XML inhibited autophagy as evidenced by the decreased accumulation of Beclin1 and autophagy related 7 (Atg7), which are necessary to form autophagosome structures. Protein kinase B (PKB/Akt), phosphatidylinositol 3 kinase (PI3K) and B cell lymphoma2 (Bcl2) levels were up-regulated, while significantly decreased protein levels for phosphorylated P38 and extracellular regulated protein kinases $1 / 2($ Erk1/2) were observed in the XML treated rats. The autophagy related results suggested that the increase in PI3K/Akt levels and inhibition of the phosphorylation of P38 MAPK and Erk1/2 contributed to the anti-autophagic activity of XML.

Conclusions: Our data suggest that XML may be effective for mitigating epirubicin-induced cardiomyopathy and inhibits autophagy via activating the PI3K/Akt signaling pathway and inhibiting the Erk1/2 and P38 MAPK signaling pathways.

\section{Keywords:}

Cardiovascular; Pharmacodynamics; Periplaneta Americana; Epirubicin; Cardiotoxicity; Xinmailong; 


\section{Graphical abstract}

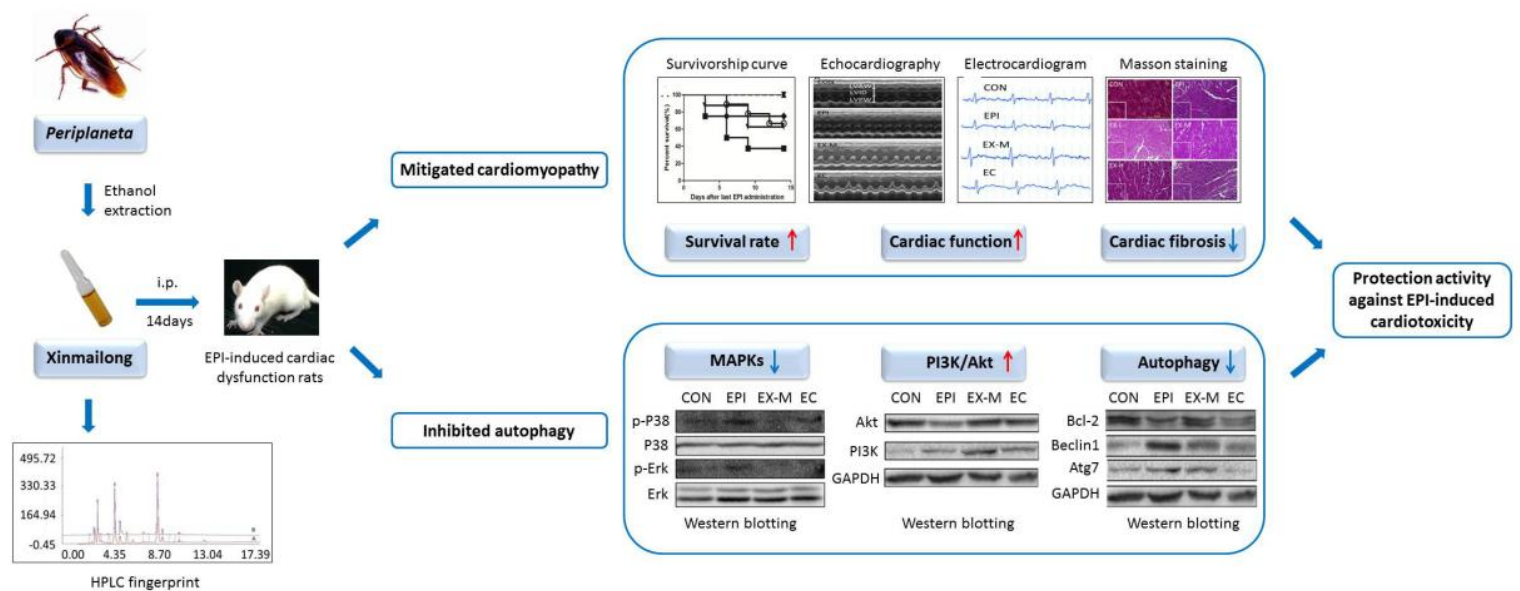




\section{Introduction}

Anthracyclines are widely used for the chemotherapeutic treatment of various types of solid tumors and hematologic malignancies (Cersosimo and Hong, 1986; Piccart et al., 2001), however, these compounds also have serious side effects including cardiomyopathy and congestive heart failure (CHF) that limit their long term administration (Wang et al., 2015a). Epirubicin (EPI) is a novel anthracycline that proved superior to Doxorubicin (DOX) in terms of activity and cardiac tolerability (Cersosimo \& Hong, 1986; Piccart et al., 2001). EPI is a semisynthetic derivative of DOX obtained by an axial-to-equatorial epimerization of the hydroxyl group at C-4' in daunosamine. This positional change has little effect on the mode of action and spectrum of activity of EPI compared with DOX, but it introduces pharmacokinetic and metabolic changes, such as a shorter terminal half-life (Danesi et al., 2002), which results in reduced cardiotoxicity. EPI was soon used at cumulative doses almost double those of DOX, resulting in equal activity but did not increase cardiotoxicity (the median cumulative dose for the development of symptomatic CHF was $900 \mathrm{mg} / \mathrm{m}^{2}$ EPI compared with $492 \mathrm{mg} / \mathrm{m}^{2}$ DOX)(Ryberg et al., 1998). Replacing DOX with EPI does not eliminate the risk of developing chronic cardiotoxicity. It should also be noted that the mechanisms underlying induced cardiotoxicity for both EPI and DOX might be the same. At present there is no specific treatment for EPI related cardiomyopathy. The efficacy of digoxin, $\beta$-blockers and angiotensin-converting enzyme inhibitors on EPI induced cardiomyopathy and CHF is only temporary (Jiao et al., 2012).

Xinmailong Injection ${ }^{\circledR}$ (XML) is a bioactive composite extracted from Periplaneta (a species of cockroach), and approved by the China State Food and Drug Administration (CFDA) in 2006 for the treatment of cardiac dysfunction. Previously, the chemical constituents, quantitative analysis and characteristic fingerprint of XML have been systematically studied (Jiao et al., 2012; Jiao et al., 2011). It was reported that 29 compounds were identified using high-performance liquid chromatography (HPLC) and gas chromatograph-mass spectrometer (GC-MS) from the active fraction of XML, which included polyhydric alcohols (percentage composition: 38.5\%), organic acids (percentage composition: 18.8\%), a variety of alkaloids (including pyrroles, piperidines, piperazine, percentage composition: 6.55\%), and some micro constituents, such as divinyl sulfide, noradrenaline and ketone compounds (Jiao et al., 2012; Jiao et al., 2011). Using insects, such as the cockroach, for the treatment of disease has a long history in traditional Chinese medicine. The famous classical book from the Chinese materia medica called "Bencao Gangmu", which was written during the Ming dynasty (AD1368-AD1644), describes the cockroach as bitter in taste and cold in nature, and identified medicinal activities related to such processes as strong heart, increasing urination, improvement microcirculation, and, increasing the blood flow to the heart, brain, lung, and kidney. Some of the above mentioned uses have been supported by evidence from an increasing number of studies and clinical reports (Ma et al., 2013; Liu et al., 2014a). Xinmailong Injection (XML) shows reasonable protective effects against cardiovascular injury (Ma et al., 2013) and anthracycline induced cardiac toxicity, enhance patients' tolerance to anthracycline chemotherapy (Liu et al., 2014a). The present study aims to clarify the protective effects of XML against EPI-induced cardiotoxicity in vivo and determine its underlying mechanisms.

\section{Materials and methods}

\subsection{Chemicals}

Xinmailong Injection, prepared from Periplaneta, is mass-produced as a drug, based on national 
standards approved by CFDA. Briefly, coarse powdered Periplaneta is extracted by percolation with $90 \%$ ethanol for 48 hours. The extract is vacuum condensed and then dissolved in normal butanol. The solution is then stirred with acticarbon for 1 hour, followed by filtration and dilution with water for injection. XML used in our research was manufactured by Tengyao Medicine Pharmaceutical Co., LTD (Yunnan, China), in accordance with applicable Good Manufacturing Practice (GMP). HPLC analysis was performed to identify the chemical characteristics of XML. The fingerprint spectrum similarity between our samples and standard is up to 0.99 by Computer Aided Similarity Evaluation software, satisfying the national standards for Xinmailong Injection proved by the CFDA. An HPLC chromatogram of XML is shown in Fig. 1.

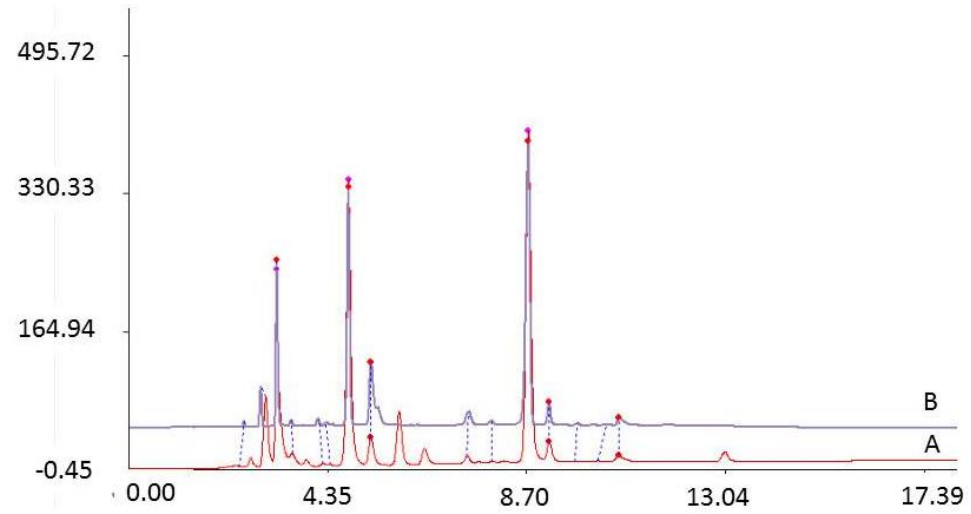

Fig. 1. HPLC fingerprint of Xinmailong Injection. (A) HPLC fingerprint of standards proved by the CFDA; (B) HPLC chromatogram of a sample of our Xinmailong Injection used in our research. The fingerprint spectrum similarity between the sample and the standard is 0.99 .

Epirubicin was obtained from Sanlian Harbin pharmaceutical Co., LTD (Harbin, China). Captopril was purchased from Sigma-Aldrich Co. (St. Louis, MO, USA).

\subsection{Animals.}

The protocol for the in vivo study with rats conforms to the Guide for the Care and Use of Laboratory Animals published by NIH. Adult male Sprague-Dawley rats, weighting 160g-180g, were purchased from the Animal Center of the Peking University Health Science Center (Beijing, China). Animals were housed under controlled environmental conditions, including temperature, humidity, and lighting. Standard laboratory chow and water were provided ad libitum.

An acute toxicity study has found that the Lethal Dose 50 (LD50) for XML intraperitoneal injection in mice is between $1117-1301 \mathrm{mg} / \mathrm{kg}$ (Liu, 2016). Sprague-Dawley rats were randomly assigned to the following groups: CON (Control, normal saline, $\mathrm{n}=8$ ), EPI (epirubicin $8 \mathrm{mg} / \mathrm{kg}$, intraperitoneal injection, n=16), EX (epirubicin + Xinmailong $125(E X-L, n=8), 250(E X-M, n=8)$ and $500(\mathrm{EX}-\mathrm{H}, \mathrm{n}=8) \mathrm{mg} / \mathrm{kg}$, intraperitoneal injection) and $\mathrm{EC}$ (epirubicin+captopril $16 \mathrm{mg} / \mathrm{kg}$, gavage, $n=8$ ). Rats were injected intraperitoneally with normal saline or a single dose of epirubicin $(8 \mathrm{mg} / \mathrm{kg})$ every five days for a total of three injections and then treated with normal saline, Xinmailong or captopril for 14 days (Fig. 2).

\begin{tabular}{|c|c|c|c|c|}
\hline & 5 days & 5 days & 5 days & 14 days \\
\hline & $\uparrow$ & $\uparrow$ & $\uparrow$ & $\uparrow \uparrow \uparrow \uparrow \uparrow \uparrow \uparrow \uparrow \uparrow \uparrow \uparrow \uparrow \uparrow \uparrow$ \\
\hline CON & NS & NS & NS & NS \\
\hline EPI & EPI & EPI & EPI & NS \\
\hline EX & EPI & EPI & EPI & $\mathrm{XML}$ \\
\hline EC & EPI & EPI & EPI & captopril \\
\hline
\end{tabular}

Fig. 2. Timeline for drug administration to rats in each group. Rats received a pre-treatment with normal saline or a 
single dose of epirubicin $(8 \mathrm{mg} / \mathrm{kg})$ every five days for a total of three injections. Pre-treatment started 15 days prior to therapy with normal saline, Xinmailong, and captopril. Xinmailong (125, 250 and 500mg/kg, intraperitoneal injection) and captopril (16mg/kg, gavage) were administered daily for a total of 14 days.

\subsection{Echocardiography}

Transthoracic echocardiography was performed on pentobarbital anesthetized rats at rest using a high-resolution imaging system for small animals (Vevo770, VisualSonics, Toronto, Canada), equipped with a high-frequent ultrasound probe (RMV-707B). Parasternal long-axis and short-axis views were acquired. Left ventricular (LV) dimensions and wall thicknesses were determined from the parasternal short axis M-mode images. Ejection fraction (EF) and fractional shortening (FS) were calculated using the Vevo770 software. The final data represent the averaged values from 3-6 cardiac cycles.

\subsection{Electrocardiographic recordings}

Surface electrocardiographic (ECG) recordings were obtained from conscious rats. For the analysis of ECGs, the onsets and offsets of the P, Q, R, S, and T waves were determined by measuring the earliest (onset) and the latest (offset) times from lead II. Since the length of the QT interval can be affected by heart rate, corrected QT (QTc) intervals were calculated by dividing the QT interval in seconds by the square root of the R-R interval in seconds (Bhandare et al., 2015).

\subsection{Reverse transcription and real-time PCR}

We used quantitative real time RT-PCR to detect changes in the mRNA expression of myocardial fibrosis associated molecules. Heart tissues were homogenized and total RNA was isolated using Trizol LS reagent (Invitrogen, Carlsbad, USA) according to the manufacturer's instruction. Amplifications were performed with the BIO-RAD MiniopticonTM Real-Time PCR Detection system CFB-3120 using iQTM SYBR Green Supermix 170-8880 (Bio-Rad) with the primers listed in Table 1. Amplifications were performed using the following conditions: initial denaturation at $95^{\circ} \mathrm{C}$ for $10 \mathrm{~min}$ followed by 39 cycles performed at $95^{\circ} \mathrm{C}$ for $15 \mathrm{~s}$ and $57^{\circ} \mathrm{C}$ for $1 \mathrm{~min}$. Transcript levels were normalized to those of Gapdh.

Table 1 Oligonucleotide sequences of the specific primers used in quantitative real time RT-PCR

\begin{tabular}{|c|c|c|}
\hline Gene & Protein & primer \\
\hline \multirow{3}{*}{ Mas } & \multirow{3}{*}{ Proto-oncogene Mas } & F-5'-GTCATGTGTATTGACAGCGGAGA-3' \\
\hline & & \\
\hline & & R-5'-CATGAGCGGAGTGAAGACCA-3' \\
\hline \multirow{3}{*}{ Ace 2} & \multirow{3}{*}{ Angiotensin-converting enzyme 2} & F-5'-AATCGTAGGCTCTGGGCTTGG-3' \\
\hline & & \\
\hline & & R-5'-TTCGATCAACTGGTTTCGGTTGTA-3' \\
\hline \multirow{3}{*}{ Ace } & \multirow{3}{*}{ Angiotensin-converting enzyme } & F-5'-TGCCTCAGCCTGGGACTTCTA-3' \\
\hline & & \\
\hline & & R-5'-CCCATTTCGTGGTGGGCTA-3' \\
\hline \multirow{3}{*}{ Agtrl } & \multirow{3}{*}{ Type-1A angiotensin II receptor } & F-5'-TGGCGATTGTGCTTTTCTTC-3' \\
\hline & & \\
\hline & & R-5'-CAGAGGGTTCAGGCAGTTGTT-3' \\
\hline \multirow{3}{*}{$T g f b 1$} & \multirow{3}{*}{ Transforming growth factor beta- 1} & F-5'-CATTGCTGTCCCGTGCAGA-3' \\
\hline & & \\
\hline & & R-5'-AGGTAACGCCAGGAATTGTTGCTA-3' \\
\hline
\end{tabular}




\begin{tabular}{|c|c|c|}
\hline \multirow{3}{*}{ Mmp2 } & \multirow{3}{*}{$72 \mathrm{kDa}$ type IV collagenase } & F-5'-ACACCCTCAAGAAGATGCAGAAG-3' \\
\hline & & \\
\hline & & R-5'-CGGAAAGAAGTTGTAGTTGG-3' \\
\hline \multirow{3}{*}{ Mmp9 } & \multirow{3}{*}{ Matrix metalloproteinase- 9} & F-5'-CCCACTTACTTTGGAAACG-3' \\
\hline & & \\
\hline & & R-5'-GAAGATGAATGGAAATACGC-3' \\
\hline \multirow{3}{*}{ Timp4 } & \multirow{3}{*}{ Metalloproteinase inhibitor 4} & F-5'-CTGGAGGGGCTGTTTGTTTC-3' \\
\hline & & \\
\hline & & R-5'-TATGTCAGCGGGGTCTT-3' \\
\hline \multirow{2}{*}{ Gapdh } & Glyceraldehyde-3-phosphate & F-5'-ACAAAGTGGACATTGTTGCC-3' \\
\hline & dehydrogenase & R-5'-AAACATGGTGGTGAAGACGC-3' \\
\hline
\end{tabular}

\subsection{Western blot analysis}

Heart tissue was homogenized and protein quantified using the Bradford method. Sixty micrograms of protein was separated on SDS-PAGE, with the separated proteins transferred by electroelution onto PVDF paper (Millipore, Billerica, USA). To clarify how XML regulated myocardial cell autophagy and autophagy-related pathway, we probed blots with rabbit antibodies to B cell lymphoma2 (Bcl2), Bcl-2 assaciated $\mathrm{X}$ protein (Bax), protein kinase B (PKB/Akt), phosphatidylinositol 3 kinase (PI3K) , extracellular regulated protein kinases 1/2 (Erk1/2), phospho-Erk1/2 (Thr202/Tyr204), P38, phospho-P38(Thr180/Tyr182), Beclin1, and autophagy related 7 (Atg7). All antibodies were obtained from Cell Signaling Technology (Beverly, MA, USA) and were used at 1:1000 dilution with incubation overnight at $4^{\circ} \mathrm{C}$. Antibody binding was detected after incubation with appropriate secondary antibodies conjugated with horse radish peroxidase, with the membrane-bound antibodies visualized by luminescence Chemi Doc XRS (Bio-Rad, Hercules, CA, USA).

\subsection{Masson staining}

Mouse cardiac tissue was first fixed with Bouin's fixative and stained with Weigert's hematoxylin and Biebrich scarlet-acid fuchsin (Sigma, Milwaukee, USA), followed by treatment with phosphomolybdic-phosphotungstic acid solution (Sigma) and staining by methylblue and Mayer's Hematoxylin and Eosin. The extent of fibrosis in the myocardial tissue sections was quantified using Image-Pro Plus 6 (Media Cybernetics, Rockville, USA) as the relative area of positive stained area (blue-green fibrosis) normalized to the total tissue area.

\subsection{Statistical Analysis}

Results are shown as means \pm SD. Differences between the control and experimental groups were evaluated by one-way (ANOVA; SPSS 13.0 for Windows, SPSS Inc., Chicago, IL, USA). $P$ values less than 0.05 were considered to be statistically significant.

\section{Results and Discussion}

Epirubicin is a novel anthracycline that has proved to be superior to DOX in terms of activity and cardiac tolerability, thus epirubicin is more valuable for the treatment of various types of cancers than DOX (Minotti et al., 2004). DOX-induced cardiotoxicity has a cumulative effect, and its underlying mechanisms are closely associated with DOX-mediated lethal oxidative stress, necrosis, apoptosis and autophagy in myocardial cells (Minotti et al., 2004; Wang et al., 2014). The mechanisms underlying the induced cardiotoxicity of EPI and DOX might be same. Due to our incomplete 
understanding of the multi-factorial cellular and molecular drivers underlying epirubicin cardiotoxicity, optimal therapeutic approaches for the protection against EPI cardiotoxicity have not yet been identified (Xi et al., 2012). Therefore, there is an ongoing and urgent need to search for a better and safer cardio protectant against EPI toxicity.

Xinmailong Injection, a bioactive composite extracted from Periplaneta americana, can dilate coronary arteries, antagonize the activation of neuroendocrine systems, increase blood supply to cardiac muscle, improve cardiac function, and antagonize ventricular restructuring (Liu et al., 2014b). The present study examined the possible protective effect of XML on EPI-induced heart failure in a rat model.

\subsection{XML improves cardiac function}

XML administration for 14 days has high protection activity against myocardial injury induced by EPI. This protection of XML was evident from improved survival rate, echocardiographic and electrocardiograph analysis.

\subsubsection{Influence of XML on survival rate and body weight after epirubicin treatment}

All animals were observed every day after the last epirubicin injection, with deaths recorded. EPI-induced cardiac dysfunction rats (EPI rats) had a survival rate of 50\% at 6 days, decreasing to $37.5 \%$ at 9 days and remaining steady at this rate until 14 hours after the last EPI administration. In contrast, CON (control) and EX-M (EPI-induced cardiac dysfunction rats treated with $250 \mathrm{mg} / \mathrm{kg}$ XML) rats presented a 100\% survival rate throughout the XML treatment period of 14 days. Similarly, Rats treated with $125 \mathrm{mg} / \mathrm{kg}$ XML disclosed a survival rate of $87.5 \%$ at 3 days, decreasing to $62.5 \%$ at 9 days and remaining steady at this rate until 14 days after EPI. Rats treated with $500 \mathrm{mg} / \mathrm{kg}$ XML disclosed a survival rate of $75 \%$ at 6 days and remaining steady at this rate until 14 days after EPI (Fig. 3A).

Epirubicin caused a statistically significant $(P<0.001)$ reduction in the increase in body weight of the rats compared to the CON group (Fig. 3B). No statistically significant difference in weight was observed among the treatment groups (Fig. 3B). These results showed that while XML improved the survival rate, it did not have a significant influence on the body weight of the rats. The improved survival rate suggested that XML plays an important protection role in the development of DOX-induced cardiomyopathy.
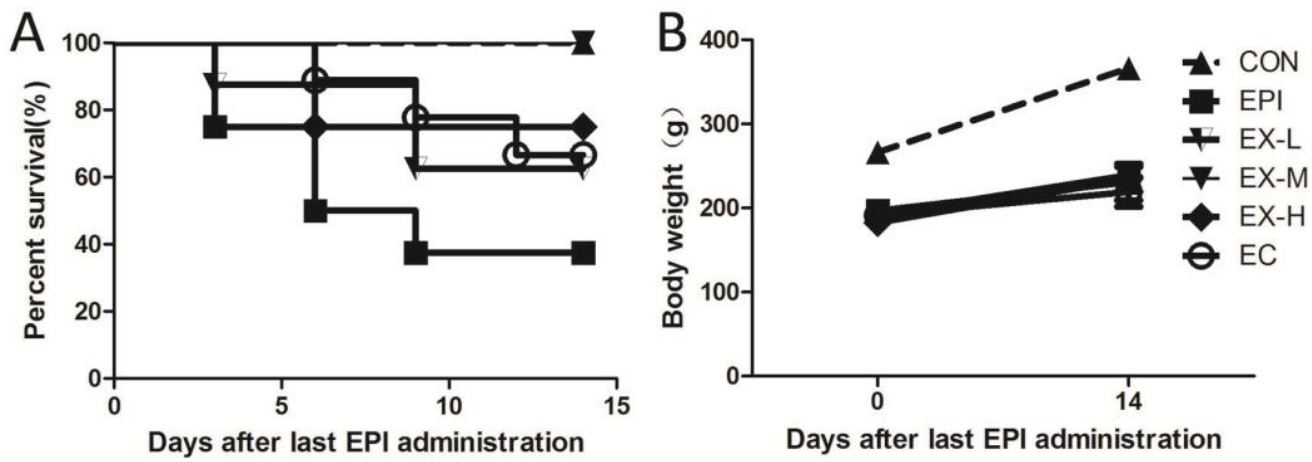

Fig. 3. Survival and weights of rats after XML treatment. All animals were observed every day after the last epirubicin injection, with deaths and weight recorded. (A) Percent survival at each day after the last epirubicin injection. No deaths occurred in the CON and EX-M groups, while the survival rates for the EPI, EX-L, and EX-H groups were $37.5 \%, 62.5 \%$, and $75 \%$, respectively. There were 16 rats in EPI group, as well as 8 rats in other groups. (B) Average body weight at days 0 and 14 after the last epirubicin injection. CON (Control, normal saline), EPI (epirubicin 8mg/kg, intraperitoneal injection), EX (epirubicin + Xinmailong 125 (EX-L), 250 (EX-M) and 500 (EX-H) 
$\mathrm{mg} / \mathrm{kg}$, intraperitoneal injection) and EC (epirubicin + captopril 16mg/kg, gavage).

\subsubsection{Influence of XML on left ventricle wall thickness and the ejection fraction}

Thinning and dilatation of the ventricular walls and a reduction in the ejection fraction are characters of anthracycline-induced heart failure (Prysyazhna et al., 2016). In the present study, Doppler and M-mode images revealed that significant echocardiographic changes are found in epirubicin-induced heart failure rats. Left ventricle (LV) posterior wall thickness (LVPW;s) and LV anterior wall thickness during systole (LVAW;s) were significantly decreased in the EPI group, compared to CON group, which was significantly ameliorated by XML treatment (Fig. 4B and 4D). Epirubicin also significantly reduced the ejection fraction $(\% \mathrm{EF})$ and fraction of shortening $(\% \mathrm{FS})$ compared to the CON group, which was also significantly ameliorated by XML (Fig. 4H and 4I). Moreover, epirubicin administration elevated left ventricle volume during systole (LV Vol;s) compared to the CON group, which was also significantly ameliorated by XML (Fig. 4F).

Our data indicated that administration of XML for 14 days yielded a high resistance to EPI-induced myocardial injury. This protection by XML was evident from echocardiographic analysis of left ventricular wall thickness and cardiac function. Similar changes in cardiac tissue have been reported in patients with chronic congestive heart failure (Liu et al., 2014b; Ma et al., 2013). Thus, XML consistently the improved survival rate of rats treated with XML to EPI-induced cardiac dysfunction (Fig. 3A) is strongly linked to increased ejection fraction and ameliorated ventricular walls thickness.
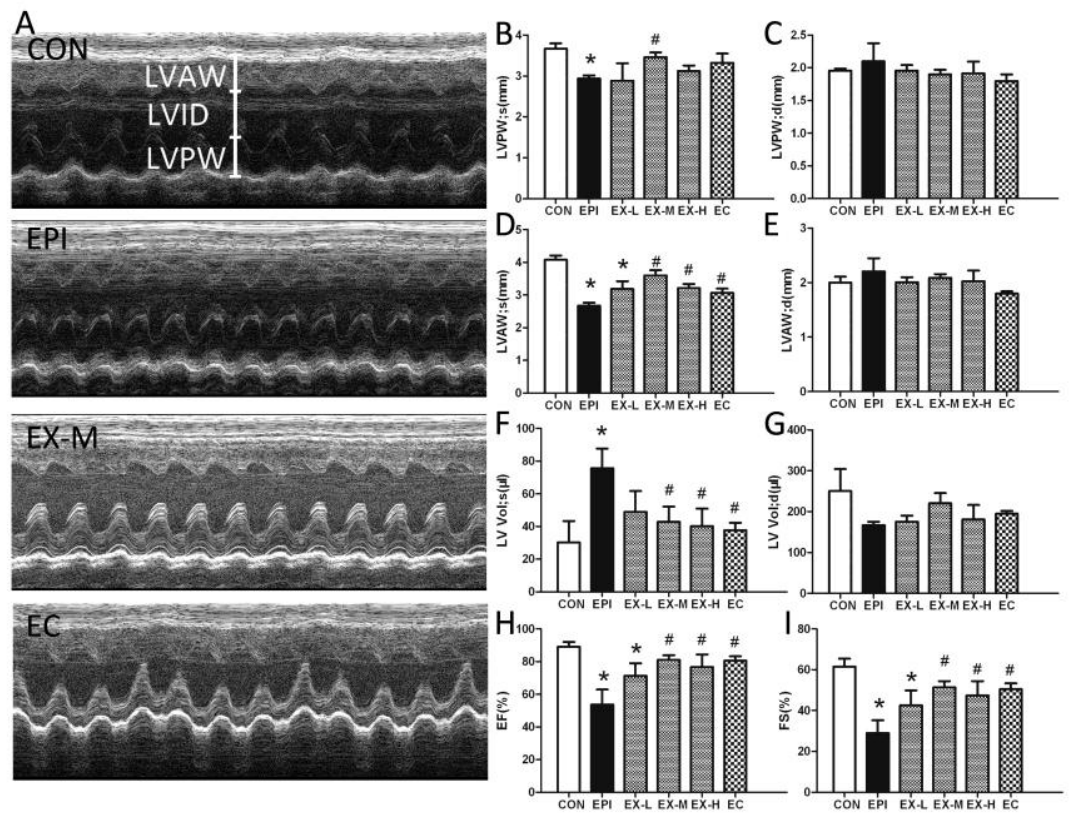

Fig. 4. Xinmailong prevents epirubicin-induced cardiac dysfunction in SD rats. (A) Echocardiography of SD rats. M-Mode echocardiography indicates that the EPI treatment decreased Left ventricle (LV) posterior wall thickness (LVPW;s), LV anterior wall thickness during systole (LVAW;s), the left ventricular fractional shortening (FS) and left ventricular ejection fraction (EF), but that XML has a protective effect. (B-I) echocardiography parameters were compared among the 6 groups. CON (Control, normal saline), EPI (epirubicin 8mg/kg, intraperitoneal injection), EX (epirubicin + Xinmailong 125 (EX-L), $250(\mathrm{EX}-\mathrm{M})$ and $500(\mathrm{EX}-\mathrm{H}) \mathrm{mg} / \mathrm{kg}$, intraperitoneal injection) and EC (epirubicin + captopril $16 \mathrm{mg} / \mathrm{kg}$, gavage). Values are expressed as mean \pm SEM $(\mathrm{n}=3)$. Asterisk $(*)$ refers to statistical significance $(P<0.05)$ in comparisons with CON group, while \# refers to comparisons with EPI group.

\subsubsection{Influence of XML on Surface Electrocardiograph}


Electrocardiograph (ECG) abnormalities are the main criteria generally used for a definite diagnosis of myocardial injury. Our study showed a significant alteration in the ECG patterns in EPI-administered rats compared to normal CON rats. Surface ECG recordings showed significantly longer QT intervals and corrected QT (QTc) intervals in the EPI rats, as did QRS duration. Changes in heart rates were not statistically different among the six groups. Similar changes in DOX-induced cardiotoxicity have been reported by other investigators (Ozkanlar et al., 2014). However, these changes are nonspecific and can represent several acute and chronic processes; in addition, all of these changes can represent "normal variants". But, if we consider the results of the control group, it is reasonable to hypothesize that EPI caused the acute changes seen in the hearts of our animals. The abnormal ECGs observed in Fig. 5A could be due to accumulating damage to cells in the injured myocardium. Treatment of the EPI rats with XML significantly recovered these changes, such as, QT, QTc intervals and QRS duration (Fig. 5). These findings suggest that the protective effect of XML involves reducing cardiac damage and susceptibility to EPI in these animals.
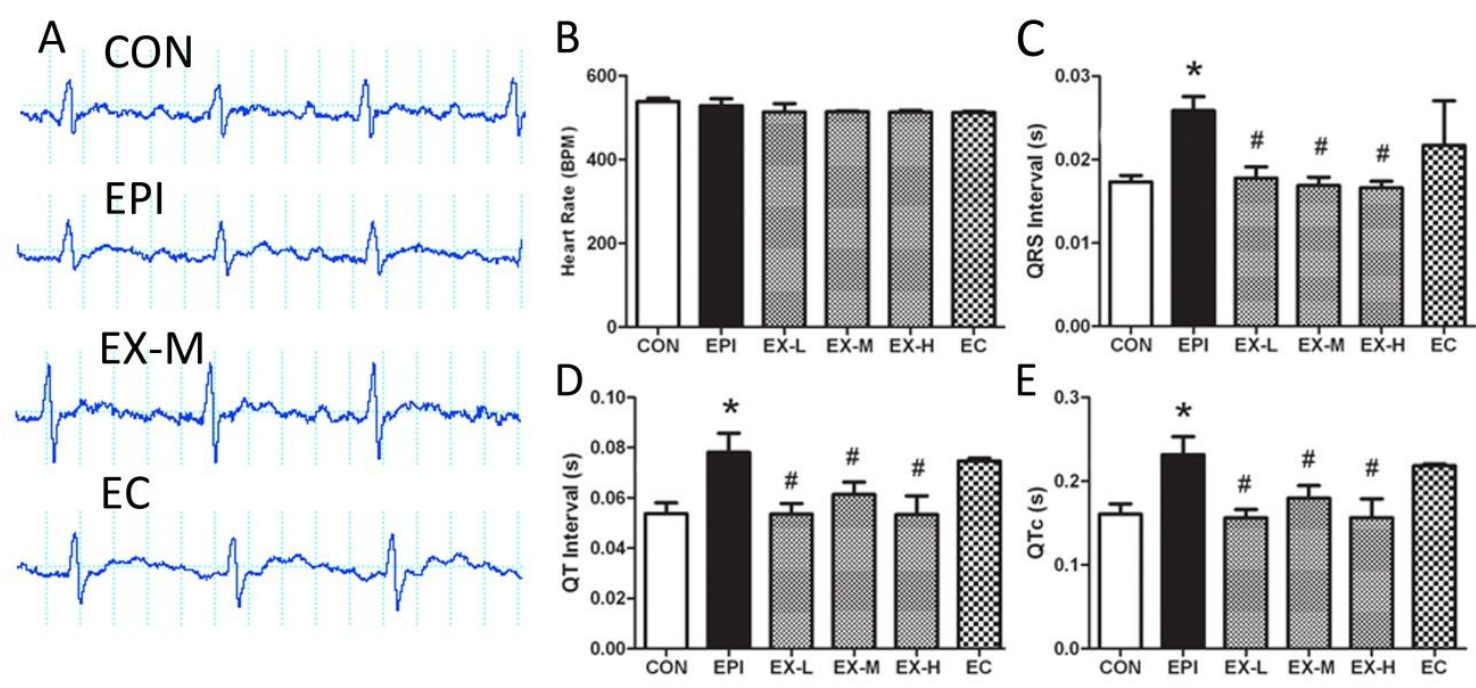

Fig. 5. Xinmailong improves the electrophysiology parameters as measured by a surface electrocardiogram. (A) electrocardiogram recording of SD rats. Treatment of EPI rats with XML significantly shortened both the QT, QTc intervals and QRS duration. (B-E) electrocardiogram parameters were compared among the 6 groups. CON (Control, normal saline), EPI (epirubicin 8mg/kg, intraperitoneal injection), EX (epirubicin + Xinmailong 125 (EX-L), 250 $($ EX-M) and 500 (EX-H) mg/kg, intraperitoneal injection) and EC (epirubicin + captopril $16 \mathrm{mg} / \mathrm{kg}$, gavage). Values are expressed as mean \pm SEM $(n=6)$. Asterisk $(*)$ refers to statistical significance $(P<0.05)$ in comparisons with CON group, while \# refers to comparisons with EPI group.

\subsection{XML reduced the severity of cardiac fibrotic remodeling}

\subsubsection{Influence of XML on the accumulation of collagen}

The fibrotic response is essential for maintaining the structure of the heart and preserving cardiac function in response to injury (Meredith et al., 2015), however unresolved fibrotic remodeling can lead to increased residual interstitial fibrosis and result in myocardial stiffness, imperfect electrical propagation and myocyte disarray. Under the microscope, significantly increased levels of myocardial collagen was observed in epirubicin-treated rats, which was broken and arranged in a disordered collagen fiber network around the myocardial cells (Fig. 6). After XML injection, the distribution of collagen (Masson positive material) was almost uniform, where the collagen fiber network among the adjacent cells was intact and had lower levels of collagen fiber content (Fig. 6). The content of collagen in the myocardial tissue was measured and when compared with control rats, 
the collagen content of the EPI rats was significantly increased, suggesting the accumulation of collagen. Compared with EPI rats, the collagen content in the XML treated groups was significantly decreased, suggesting that XML inhibited the accumulation of collagen (Fig. 6).
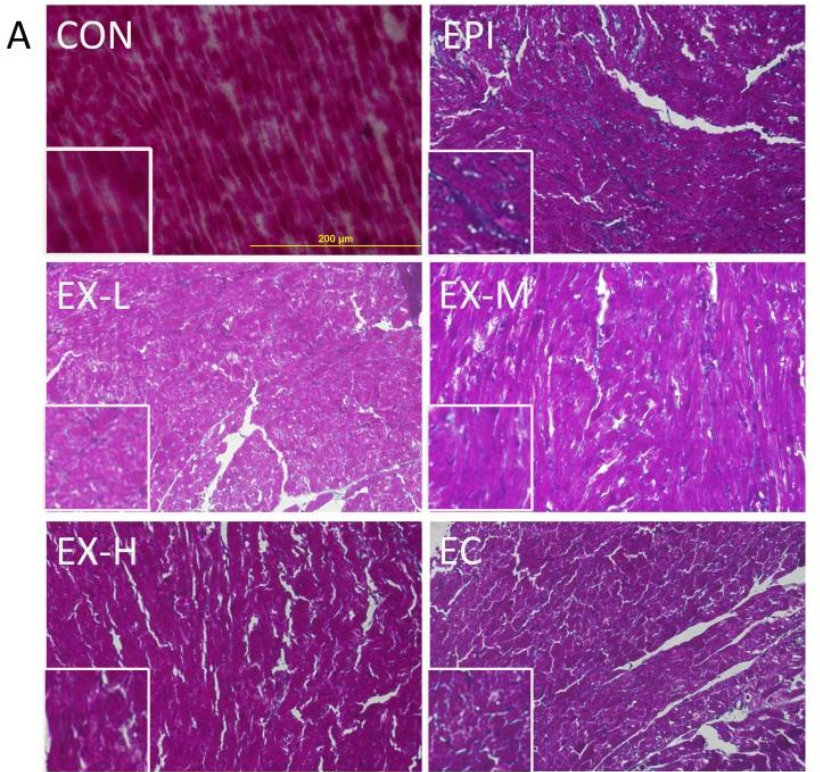

B

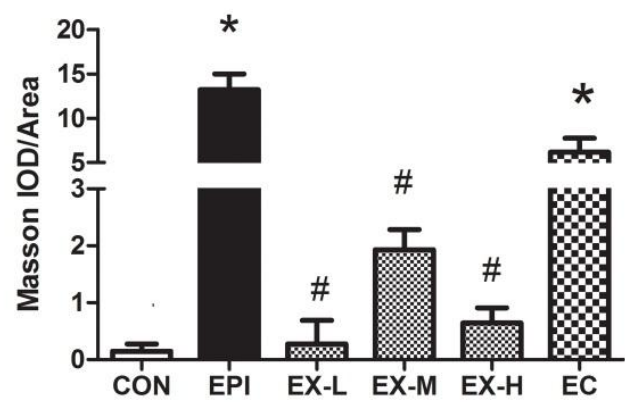

Fig. 6. Xinmailong prevents cardiac fibrosis. Cardiac fibrosis levels were investigated by Masson staining. (A) Masson staining was performed to evaluate collagen deposition in left ventricular (LV) tissue. (B) Collagen volume fraction (positive Masson IOD/area) was measured using the Image-Pro Plus 6.0 software. Less collagen was deposited in Xinmailong-treated rats. CON (Control, normal saline), EPI (epirubicin 8mg/kg, intraperitoneal injection), EX (epirubicin + Xinmailong 125 (EX-L), 250 (EX-M) and 500 (EX-H) mg/kg, intraperitoneal injection) and EC (epirubicin + captopril $16 \mathrm{mg} / \mathrm{kg}$, gavage). Values are expressed as mean \pm SEM. Asterisk $(*)$ refers to statistical significance $(P<0.05)$ in comparisons with CON group, while \# refers to comparisons with EPI group.

\subsubsection{Influence of XML on the expression of Mmps and Timp4 mRNAs}

The expression of Mmp2, Mmp9, as well as Timp4 in the myocardium was detected by quantitative real time RT-PCR. Expression levels of Mmp2, Mmp9, and Timp4, including their expression ratios, were analyzed. Mmp9 mRNA levels were significantly $(P<0.05)$ increased upon EPI treatment, with no significant change seen for Mmp2 or Timp4 (Fig. 7). XML treatment restored Mmp9 mRNA levels, had no effect on $M m p 2$, but caused a significant $(P<0.05)$ elevation in Timp4 mRNA levels (Fig. 7). MMP-9, coded by the Mmp 9 gene, is responsible for degrading extracellular matrix components that play a role in ventricular dilation (Voorhees et al., 2015). MMP-9 activity is regulated at the transcriptional and translational level and by TIMPs at the posttranslational level (Mani et al., 2015). As the activity of MMPs is dependent upon the relative abundance of the MMPs compared to their inhibitors (TIMPs), we examined the Mmp2/Timp4 and Mmp9/Timp4 mRNA ratios. No significant 
difference in the Mmp2/Timp4 ratio was observed between groups, despite the significant $(P<0.05)$ increase in Timp4 expression in XML treatment, while the significant $(P<0.05)$ increase in the Mmp9/Timp4 ratio with EPI treatment was reversed after the XML treatment (Fig. 7).

Anthracycline has been reported to induce an increase in MMPs, which could be involved in collagen degradation (Polegato et al., 2015). In this study, the EPI group presented increased Mmp9 mRNA expression with thinned LV wall thickness and enlarged left ventricle volume. These results indicate that $M m p$ might play a role in EPI-induced cardiac fibrotic remodeling. It is therefore probable that decreased Mmp9 mRNA expression and Mmp-9/Timp-4 mRNA ratios results in a decrease in ventricular dilation and subsequent improvement of cardiac function in XML treated rats.

\subsubsection{Influence of XML on the expression of $\mathrm{Tg} f b 1 \mathrm{mRNAs}$}

TGF- $\beta 1$, coded by the $T g f b 1$ gene, contributes to cardiac fibrosis development, hypertrophy and apoptosis (Lin et al., 2015). In the present study, Tgfbl mRNA levels were significantly $(P<0.05)$ increased upon EPI treatment and restored with XML treated. (Fig. 7A) EPI-induced cardiotoxicity is thought to be a complex multifactorial process, which includes up-regulated TGF- $\beta$ signaling. The TGF- $\beta$ signaling pathways play important roles in collagen synthesis (Meng et al., 2015). Inhibition of TGF- $\beta$ signaling alleviates left ventricular remodeling, and systolic and diastolic dysfunction. Our data found that XML alleviated the accumulation of collagen with decreased Tgfbl gene expression. These actions of XML might therefore be responsible, at least in part, for the attenuation of systolic and diastolic cardiac dysfunction.
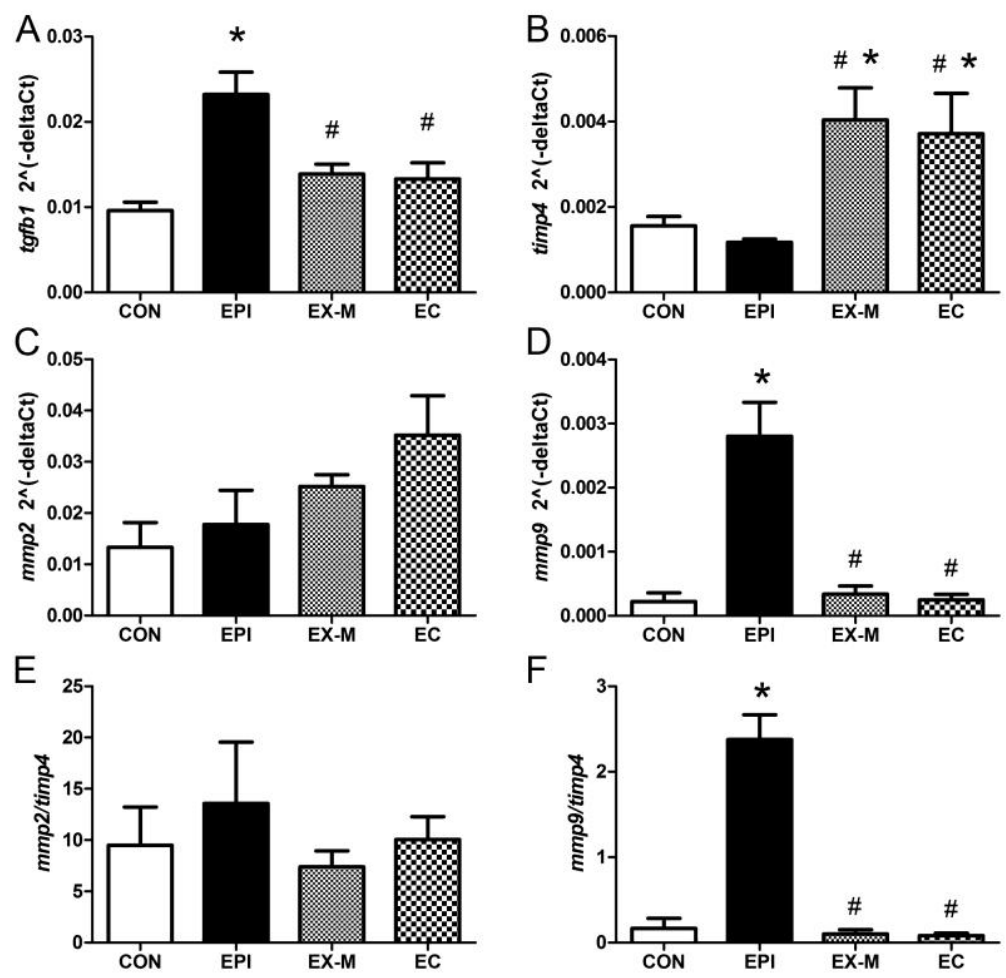

Fig. 7. Influence of XML on mRNA levels for Mmps and $T g f b 1$. Xinmailong suppressed the mRNA levels of $T g f b 1$ (A) and Mmp9 (D), and promoted expression of Timp4 (B) with a decrease in the ratio of Mmp9/Timp4 (F). The relative amount of mRNA in the myocardial was determined by real-time PCR and corrected to Gapdh as an internal standard. CON (Control, normal saline), EPI (epirubicin $8 \mathrm{mg} / \mathrm{kg}$, intraperitoneal injection), EX-M (epirubicin + Xinmailong 250mg/kg, intraperitoneal injection) and EC (epirubicin + captopril $16 \mathrm{mg} / \mathrm{kg}$, gavage). Values are expressed as mean $\pm \operatorname{SEM}(n=3)$. Asterisk $(*)$ refers to statistical significance $(P<0.05)$ in comparisons with CON group, while \# refers to comparisons with EPI group. 


\subsubsection{Influence of XML on the expression of Ace, Ace2, Mas, and Agtr1 mRNAs}

ACE2 (coded by the Ace2 genes) regulate the renin-angiotensin-aldosterone system through the degradation of angiotensin (Ang) II and the formation of Ang-(1-7). Ang II induced Mmp transcription attenuates the pathogenesis of hypertension, heart dysfunction, and cardiac fibrosis (Kuan et al., 2013) via binding to its specific type-1 angiotensin II receptor (AT1, coded by the Agtrl gene), while Ang-(1-7) counteracts the effects of Ang II via its G protein-coupled receptor Mas (coded by Mas).

To examine changes in the mRNA level for Ace, Ace2, Mas and Agtrl, we extracted RNA from myocardial tissue and conducted quantitative real time RT-PCR. Quantitative real time RT-PCR results showed that only the Ace 2 mRNA expression level was significantly changed after EPI treatment, with a significant $(P<0.05)$ decrease, which was reversed upon XML treatment (Fig. 8). Captopril treatment generated a different pattern from that of XML, with significant $(P<0.05)$ increases for Ace mRNA levels, and significant $(P<0.05)$ decreases in the mRNA levels for the receptor Agtrl and Mas mRNA expression levels (Fig. 8). These findings indicate that XML downregulated Ace 2 mRNA expression, and might weaken the function of angiotensin II to induce Mmp9 gene expression, and thus attenuate the pathogenesis of heart dysfunction and cardiac fibrosis.
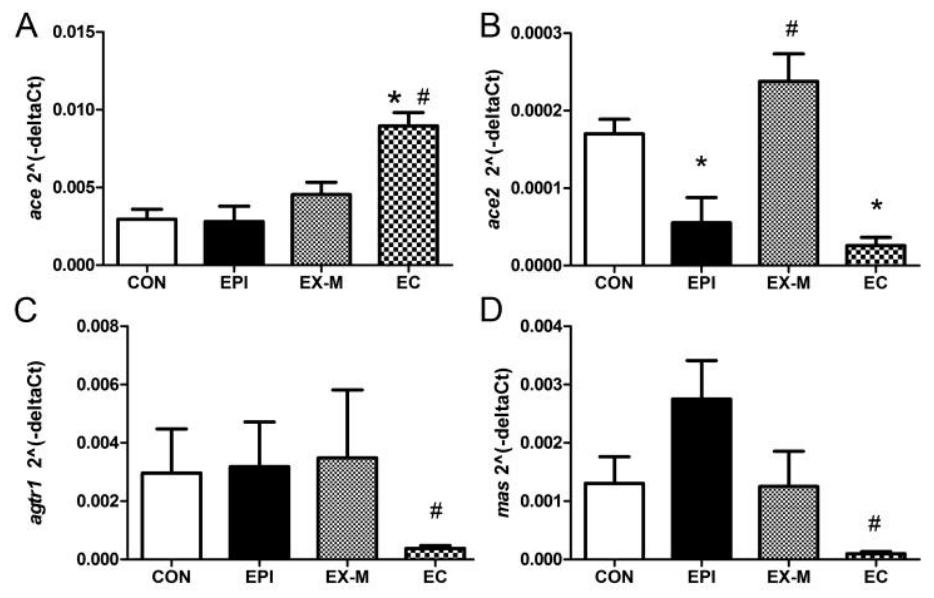

Fig. 8 Influence of XML on the mRNA levels of Ace, Ace2, Agtr1, and Mas. EPI treatment yielded significantly reduced Ace 2 (B) mRNA expression, but no significant change in Ace (A), Agtrl (C) or Mas (D). XML reversed the decrease in Ace2 (B) mRNA levels without significantly changing Ace (A), Agtrl (C), or Mas (D) levels. The relative amount of mRNA in the myocardial was determined by real-time PCR and corrected to Gapdh as an internal standard. CON (Control, normal saline), EPI (epirubicin $8 \mathrm{mg} / \mathrm{kg}$, intraperitoneal injection), EX-M (epirubicin + Xinmailong $250 \mathrm{mg} / \mathrm{kg}$, intraperitoneal injection) and EC (epirubicin + captopril $16 \mathrm{mg} / \mathrm{kg}$, gavage). Values are expressed as mean \pm SEM $(\mathrm{n}=3)$. Asterisk $(*)$ refers to statistical significance $(P<0.05)$ in comparisons with CON group, while \# refers to comparisons with EPI group.

\section{3 XML mitigated autophagy via inactivated MAPKs.}

\subsubsection{Influence of XML on autophagy}

A rapidly expanding body of evidence supports the notion that cardiac myocyte death by apoptosis, autophagy and necrosis are the primary mechanisms of doxorubicin-induced cardiotoxicity (Lee et al., 2013). Most in vitro studies suggest that DOX increases autophagy and that it likely plays a pathological role in cardiotoxicity (Dirks-Naylor, 2013). Autophagy in the myocardium has long been viewed as a double-edged sword, generally protective in nature, but also being destructive and leading to autophagic cell death (Sishi et al., 2013). Dysregulation of autophagy may also play a 
contributing role to EPI-induced cardiotoxicity.

To clarify how XML regulated myocardial cell autophagy, the protein levels for B cell lymphoma2 (Bcl2), Bcl-2 associated X protein (Bax), Beclin1 and autophagy related 7 (Atg7) were measured. Expression of Beclin1 and Atg7 protein in the myocardium were significantly $(P<0.05)$ higher in the EPI group, compared to the CON group $(P<0.05)$ (Fig. 9). Changes in Beclin1 were reversed by treatment with XML $(P<0.05)$. Our results indicated that EPI induced autophagy as evidenced by the increasing accumulation of Atg7 and Beclin1 was mitigated by the XML treatment.

Disruption of the Beclin1 complex by Bcl2 is an essential switch that turns on cellular autophagy in response to environmental stress (Marquez and $\mathrm{Xu}, 2012$ ). Recently, it has been proposed that Bcl2 may also inhibit autophagy indirectly through a mechanism dependent on the proapoptotic Bcl2 family member Bax (Pedro et al., 2015). In the present study, EPI treatment induced a decrease in the protein levels for Bcl2 $(P<0.05)$, while expression of Bax was not significantly changed. The expression of Bcl-2 was significantly restored in XML treated cardiomyopathy rats (Fig. 9). These results indicate a role for XML in maintaining a complex of Beclin1 with Bcl2 through a Bax-independent mechanism; thus, XML can turn off autophagy in the EPI-induced cardiomyopathy rat model. The present study indicates the potent anti-autophagic effects of XML.

\subsubsection{Influence of XML on the expression of PI3K and Akt}

Since the phosphatidylinositol 3 kinase (PI3K)/ protein kinase B (PKB/Akt) signaling pathway plays an essential role in mediating myocardial cell autophagy (Wang et al., 2015b), we explored whether there were any changes in this signaling pathway. Treatment with EPI significantly $(P<0.05)$ attenuated the protein levels for Akt (Fig. 9). Moreover, the EPI-induced attenuation of Akt was reversed by XML. For PI3K, no significant change was observed for the EPI group, but this level became significantly $(P<0.05)$ increased after treatment with XML (Fig. 9).

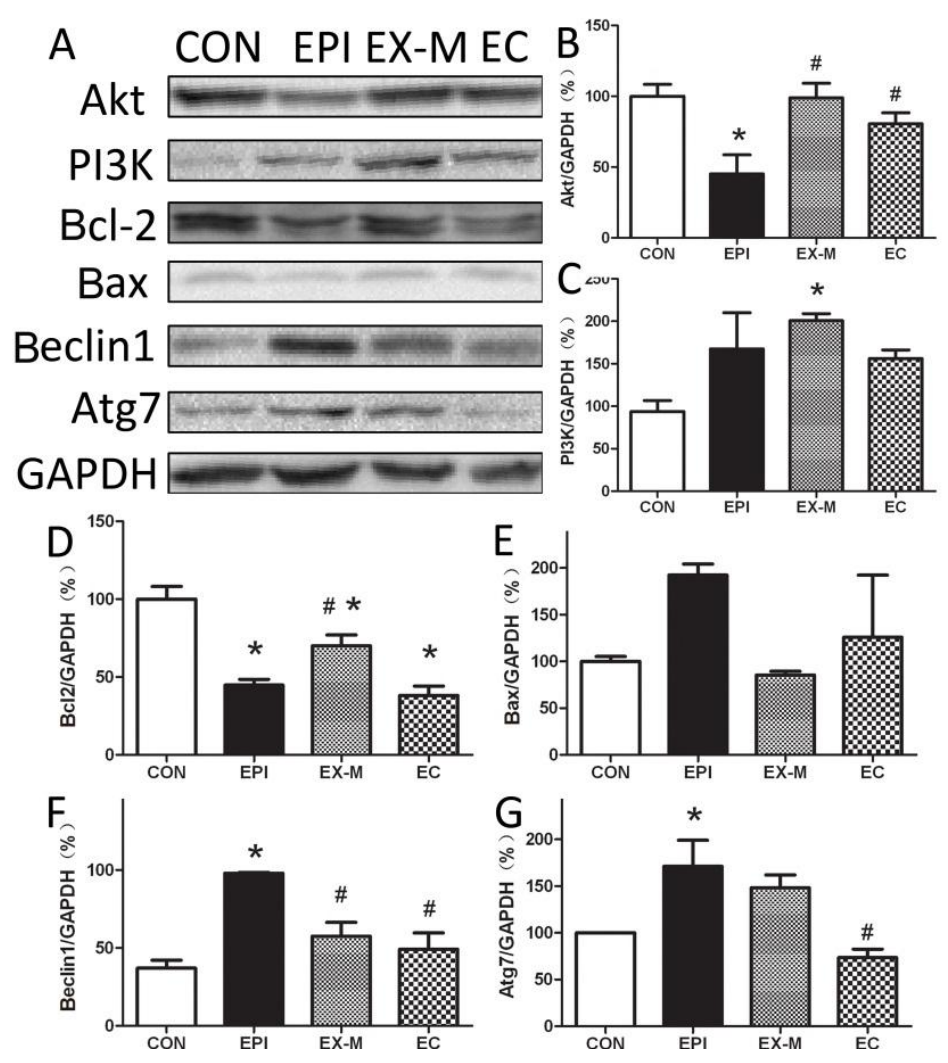

Fig. 9. Xinmailong attenuated autophagy in EPI-induced cardiac dysfunction rats. (A) Representative Western blots 
images of Akt, PI3K, Bcl2, Bax, Beclin1 and Atg7. Xinmailong enhanced Akt and PI3K expression and attenuated expression of Beclin1 and Atg7 expression, with increase in Bcl2. (B-G) Quantification of protein levels. CON (Control, normal saline), EPI (epirubicin $8 \mathrm{mg} / \mathrm{kg}$, intraperitoneal injection), EX-M (epirubicin + Xinmailong $250 \mathrm{mg} / \mathrm{kg}$, intraperitoneal injection) and EC (epirubicin + captopril $16 \mathrm{mg} / \mathrm{kg}$, gavage). Values are expressed as mean \pm SEM $(\mathrm{n}=3)$. Asterisk $(*)$ refers to statistical significance $(P<0.05)$ in comparisons with CON group, while \# refers to comparisons with EPI group.

\subsubsection{Influence of XML on the phosphorylation of P38 MAPK and Erk1/2}

The mitogen-activated protein kinases (MAPKs) are a family of serine-threonine kinases that act as key signaling molecules regulating numerous cellular functions. Extracellular regulated protein kinases 1/2 (Erk1/2) and P38 are major subfamilies of MAPKs (Jeon et al., 2015). MAPK signaling pathways are also involved in many other cellular processes including cell growth, proliferation, autophagy and apoptosis (Rovida and Stecca, 2015; Zhou et al., 2015). To examine whether these MAPK subfamilies are affected by XML, the levels of phosphorylated P38 and Erk1/2 were examined by Western blotting. Protein levels of p-P38 and p-Erk1/2 were significantly up-regulated in the EPI group, compared with the control group $(P<0.05)$, but these levels were significantly ameliorated by XML treatment $(P<0.05)$ (Fig. 10).

Taken together, these results clearly indicate that decreased activation of P38 and Erk1/2 are involved in the protective effect of XML against EPI-induced cardiotoxicity. Decreases in P38 and Erk1/2 phosphorylation increased Akt and PI3K expression and decreased the expression of Beclin1 and Atg7, which are necessary to form the autophagosomal structures. Moreover, the increase in Bcl2 inhibited Beclin1 activity also prevented mitochondria-induced apoptosis. The protective activity of XML in EPI-induced cardiotoxicity is due to the suppression of autophagy and apoptosis via up-regulation of the PI3K/Akt cascade with inactivated MAPKs. In addition, transcriptional activity is an important regulatory point for myocardial MMP abundance. The formation and activation of transcription factors in myocardial cells involves the MAPK signaling pathway (Spinale, 2007). XML reduced Mmp9 transcription by inhibiting the phosphorylation of P38 MAPK and Erk1/2.

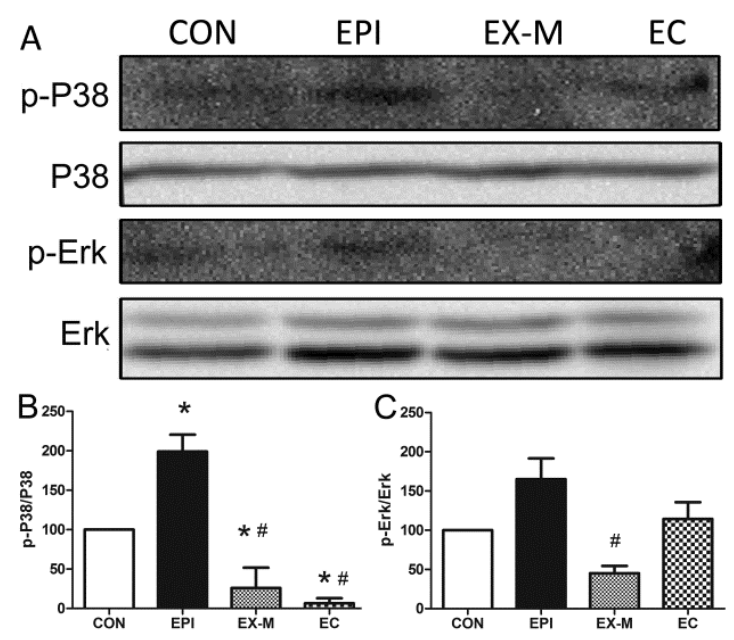

Fig.10. Xinmailong significantly attenuated P38 and Erk1/2 activities in EPI-induced cardiac dysfunction rats. (A) Phosphorylated P38 and Erk1/2 levels were measured using western blots. (B) Quantification of the levels of p-P38/P38. (C) Quantification of the levels of p-Erk/Erk. Protein levels of p-P38 and p-Erk1/2 were significantly down regulated in the Xinmailong group. CON (Control, normal saline), EPI (epirubicin $8 \mathrm{mg} / \mathrm{kg}$, intraperitoneal injection), EX-M (epirubicin + Xinmailong $250 \mathrm{mg} / \mathrm{kg}$, intraperitoneal injection) and EC (epirubicin + captopril 
$16 \mathrm{mg} / \mathrm{kg}$, gavage). Values are expressed as mean $\pm \mathrm{SEM}(\mathrm{n}=3)$. Asterisk $(*)$ refers to statistical significance $(P<$ 0.05) in comparisons with CON group, while \# refers to comparisons with EPI group.

Collectively, our results demonstrate that the protective activity of XML in EPI-induced cardiotoxicity is due to the suppression of autophagy via the up-regulation of the PI3K/Akt cascade through inactivated MAPKs. Thus, the present finding suggests that XML inhibited autophagymediated cardiotoxicity shows potential as an alternative therapeutic agent for EPI-induced heart failure (Fig. 11).

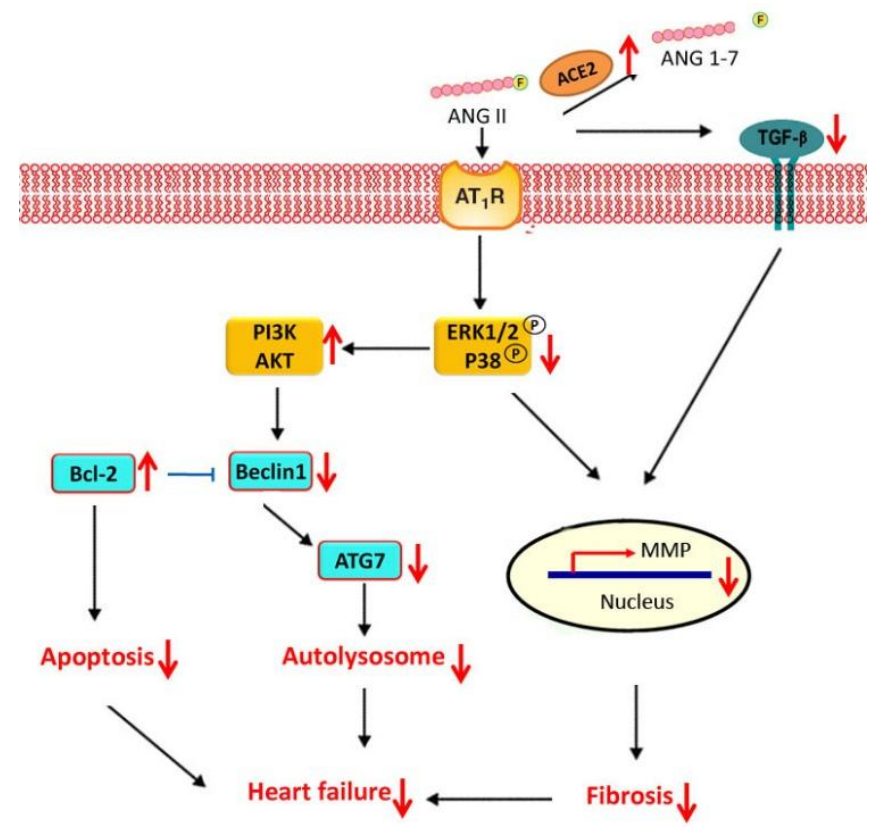

Fig.11. The schematic model of the molecular mechanisms associated with Xinmailong protective effect against EPI-induced cardiomyopathy. XML treatment augments the expression of ace 2 and suppresses the expression of $\operatorname{tg} f b 1$, which then decreases the ratio of mmp-9/timp-4 and inhibits the MAPK signaling pathway. This action of XML might therefore be responsible for the attenuation of cardiac fibrotic remodeling. On the other hand, the decrease in P38 and Erk1/2 activity increased Akt and PI3K expression and decreased the expression of Beclin1 and Atg7, which is necessary to form autophagosomal structures. Moreover, the increase in Bcl2 inhibited Beclin1 activity as well as prevents mitochondria-induced apoptosis. The protective activity of Xinmailong in EPI-induced cardiotoxicity is due to suppression of autophagy and apoptosis via up-regulation of the PI3K/Akt cascade with inactivated MAPKs.

\section{Conclusions}

In conclusion, our experiments provide evidence that XML mitigated EPI-induced cardiomyopathy and inhibited autophagy via activating the PI3K/Akt signaling pathway and inhibiting the Erk1/2 and P38 MAPK signaling pathways. Therefore, we speculate that XML may be an alternative supplementary therapeutic agent for the treatment of EPI-induced cardiomyopathy. However, the intricate relationship between autophagy and apoptosis poses a big challenge for the balance anti-cancer treatment with cardiotoxicity, hence, further studies are required to investigate above relationships by XML treatment and cardio protection effects in EPI-induced cardiomyopathy.

\section{Competing interests}


The authors declare that they have no competing interests.

\section{Acknowledgements}

This study was supported by grants from the National Natural Science Foundation of China (NSFC) Grant Number 81102484 and 30772603, National Key Technologies R\&D Program (Grant Numbers 2006BAF07B01, 2009BAK61B01, 2009BAK61B04, and 2012BAK25B01), and a grant from the National Science Foundation of China - Canadian Institutes of Health Research (NSFC-CIHR) China-Canada Joint Health Research Initiative (Grant Numbers 81061120525 and CCI-109605).

Abbreviations: ACE, angiotensin-converting enzyme; ACE2, angiotensin-converting enzyme 2; Ang, angiotensin; AT1, type-1A angiotensin II receptor; Atg7, autophagy related 7; Bax, Bcl-2 associated X protein; Bcl2, B cell lymphoma2; CHF, congestive heart failure; DOX, Doxorubicin; ECG, Surface electrocardiographic recordings; EF, ejection fraction; EPI, epirubicin; Erk1/2, extracellular regulated protein kinases 1/2; FS, fraction of shortening; HPLC, high performance liquid chromatography; LV Vol;s, left ventricle volume during systole; LVAW;d, Left ventricle anterior wall thickness during diastole; LVAW;s, Left ventricle anterior wall thickness during systole; LVPW;d, Left ventricle posterior wall thickness during diastole; LVPW;s, Left ventricle posterior wall thickness during systole; MAPK, mitogen-activated protein kinases; Mas, proto-oncogene Mas; MMP-2, 72kDa type IV collagenase; MMP-9, matrix metalloproteinases-9; PI3K, phosphatidylinositol 3 kinase; PKB/Akt, protein kinase B; QTc, corrected QT intervals; RT-PCR, reverse transcriptase polymerase chain reaction; TGF- $\beta$, transforming growth factor- $\beta$; TIMP4, metalloproteinase inhibitor 4; XML, Xinmailong Injection.

\section{References}

Bhandare, A.M., Mohammed, S., Pilowsky, P.M., Farnham, M.M., 2015. Antagonism of PACAP or microglia function worsens the cardiovascular consequences of kainic-acid-induced seizures in rats. The Journal of neuroscience 35, 2191-2199.

Cersosimo, R.J., Hong, W.K., 1986. Epirubicin: a review of the pharmacology, clinical activity, and adverse effects of an adriamycin analogue. Journal of clinical oncology 4, 425-439.

Danesi, R., Fogli, S., Gennari, A., Conte, P., Del Tacca, M., 2002. Pharmacokinetic-pharmacodynamic relationships of the anthracycline anticancer drugs. Clinical pharmacokinetics 41, 431-444.

Dirks-Naylor, A.J., 2013. The role of autophagy in doxorubicin-induced cardiotoxicity. Life sciences. PMID:24513201

Jeon, B.K., Kwon, K., Kang, J.L., Choi, Y.H., 2015. Csk-Induced Phosphorylation of Src at Tyrosine 530 is Essential for H2O2-Mediated Suppression of ERK1/2 in Human Umbilical Vein Endothelial Cells. Scientific reports 5,12725 .

Jiao, C.X., Zhang, C.G., Liu, G.M., 2012. Gas chromatography - mass spectrometry of The volatile components in the Periplaneta Americana alcohol extraction of water-soluble ingredients. Lishizhen medicine and materia research 23, 2797-2798. (in chinese)

Jiao, C.X., Zhang, C.G., Liu, G.M., Li, S.N., 2011. Establishment of HPLC fingerprint of Xinmailong Injection. Chinese Traditional Patent Medicine 33, 1648-1652. (in chinese)

Kuan, T.C., Chen, M.Y., Liao, Y.C., Ko, L., Hong, Y.H., Yen, C.Y., Hsieh, W.Y., Cheng, K.S., Wu, C.L., 
Lin, C.S., 2013. Angiotensin II downregulates ACE2-mediated enhancement of MMP-2 activity in human cardiofibroblasts. Biochemistry and cell biology 91, 435-442.

Lee, B.S., Kim, S.H., Jin, T., Choi, E.Y., Oh, J., Park, S., Lee, S.H., Chung, J.H., Kang, S.M., 2013. Protective effect of survivin in Doxorubicin-induced cell death in h9c2 cardiac myocytes. Korean circulation journal 43, 400-407.

Lin, C.Y., Hsu, Y.J., Hsu, S.C., Chen, Y., Lee, H.S., Lin, S.H., Huang, S.M., Tsai, C.S., Shih, C.C., 2015. CB1 cannabinoid receptor antagonist attenuates left ventricular hypertrophy and Akt-mediated cardiac fibrosis in experimental uremia. Journal of molecular and cellular cardiology 85, 249-261.

Liu, W., Duan, X.B., Xu, X., 2014a. Preventive effect of Xinmailong Injection on anthracyclines induced cardiac toxicity. Chinese General Practice 17, 3461-3464. (in chinese)

Liu, Y., Wang, Z., Xiao, W., Li, Y., 2014b. Use of gated myocardial perfusion imaging to assess clinical value of xinmailong injection in chronic congestive heart failure. Journal of traditional chinese medicine 34, 555-559. (in chinese)

Liu, H.X., 2016. Expert consensus about standardized application of Xingmailong. Chinese journal of integrated traditional and western medicine 36, 280-283.(in chinese)

Ma, Q., Luo, Y., Guo, P., Gao, G., Yang, M., Sablok, G., Zhang, Y., Zhou, F., 2013. Clinical effects of Xinmailong therapy in patients with chronic heart failure. International journal of medical sciences 10 , 624-633.

Mani, S.K., Kern, C.B., Kimbrough, D., Addy, B., Kasiganesan, H., Rivers, W.T., Patel, R.K., Chou, J.C., Spinale, F.G., Mukherjee, R., Menick, D.R., 2015. Inhibition of class I histone deacetylase activity represses matrix metalloproteinase-2 and -9 expression and preserves LV function postmyocardial infarction. American journal of physiology. Heart and circulatory physiology 308, H1391-1401.

Marquez, R.T., Xu, L., 2012. Bcl-2:Beclin 1 complex: multiple, mechanisms regulating autophagy/apoptosis toggle switch. American journal of cancer research 2, 214-221.

Meng, G., Zhu, J., Xiao, Y., Huang, Z., Zhang, Y., Tang, X., Xie, L., Chen, Y., Shao, Y., Ferro, A., Wang, R., Moore, P.K., Ji, Y., 2015. Hydrogen Sulfide Donor GYY4137 Protects against Myocardial Fibrosis. Oxidative medicine and cellular longevity 2015, 691070.

Meredith, A., Boroomand, S., Carthy, J., Luo, Z., McManus, B., 2015. 1,25 Dihydroxyvitamin D3 Inhibits TGFbeta1-Mediated Primary Human Cardiac Myofibroblast Activation. PloS one 10, e0128655.

Minotti, G., Menna, P., Salvatorelli, E., Cairo, G., Gianni, L., 2004. Anthracyclines: molecular advances and pharmacologic developments in antitumor activity and cardiotoxicity. Pharmacological reviews 56, 185-229.

Ozkanlar, Y., Aktas, M.S., Turkeli, M., Erturk, N., Oruc, E., Ozkanlar, S., Kirbas, A., Erdemci, B., Aksakal, E., 2014. Effects of ramipril and darbepoetin on electromechanical activity of the heart in doxorubicin-induced cardiotoxicity. International journal of cardiology 173, 519-521.

Pedro, J.M., Wei, Y., Sica, V., Maiuri, M.C., Zou, Z., Kroemer, G., Levine, B., 2015. BAX and BAK1 are dispensable for ABT-737-induced dissociation of the BCL2-BECN1 complex and autophagy. Autophagy 11, 452-459.

Piccart, M.J., Di Leo, A., Beauduin, M., Vindevoghel, A., Michel, J., Focan, C., Tagnon, A., Ries, F., Gobert, P., Finet, C., Closon-Dejardin, M.T., Dufrane, J.P., Kerger, J., Liebens, F., Beauvois, S., Bartholomeus, S., Dolci, S., Lobelle, J.P., Paesmans, M., Nogaret, J.M., 2001. Phase III trial comparing two dose levels of epirubicin combined with cyclophosphamide with cyclophosphamide, methotrexate, and fluorouracil in node-positive breast cancer. Journal of clinical oncology 19, 3103-3110. 
Polegato, B.F., Minicucci, M.F., Azevedo, P.S., Carvalho, R.F., Chiuso-Minicucci, F., Pereira, E.J., Paiva, S.A., Zornoff, L.A., Okoshi, M.P., Matsubara, B.B., Matsubara, L.S., 2015. Acute doxorubicin-induced cardiotoxicity is associated with matrix metalloproteinase-2 alterations in rats. Cellular physiology and biochemistry 35, 1924-1933.

Prysyazhna, O., Burgoyne, J., Scotcher, J., Grover, S., Kass, D., Eaton, P., 2016. Phosphodiesterase 5 Inhibition Limits Doxorubicin-induced Heart Failure by Attenuating Protein Kinase G I $\alpha$ Oxidation. Journal of Biological Chemistry. doi: 10.1074/jbc.M116.724070 (Epub ahead of print)

Rovida, E., Stecca, B., 2015. Mitogen-activated protein kinases and Hedgehog-GLI signaling in cancer: A crosstalk providing therapeutic opportunities? Seminars in cancer biology 35, 154-167.

Ryberg, M., Nielsen, D., Skovsgaard, T., Hansen, J., Jensen, B.V., Dombernowsky, P., 1998. Epirubicin cardiotoxicity: an analysis of 469 patients with metastatic breast cancer. Journal of clinical oncology $16,3502-3508$.

Sishi, B.J., Loos, B., van Rooyen, J., Engelbrecht, A.M., 2013. Autophagy upregulation promotes survival and attenuates doxorubicin-induced cardiotoxicity. Biochemical pharmacology 85, 124-134.

Spinale, F.G., 2007. Myocardial matrix remodeling and the matrix metalloproteinases: influence on cardiac form and function. Physiological reviews 87, 1285-1342.

Voorhees, A.P., DeLeon-Pennell, K.Y., Ma, Y., Halade, G.V., Yabluchanskiy, A., Iyer, R.P., Flynn, E., Cates, C.A., Lindsey, M.L., Han, H.C., 2015. Building a better infarct: Modulation of collagen cross-linking to increase infarct stiffness and reduce left ventricular dilation post-myocardial infarction. Journal of molecular and cellular cardiology 85, 229-239.

Wang, L., Tan, T.C., Halpern, E.F., Neilan, T.G., Francis, S.A., Picard, M.H., Fei, H., Hochberg, E.P., Abramson, J.S., Weyman, A.E., Kuter, I., Scherrer-Crosbie, M., 2015a. Major Cardiac Events and the Value of Echocardiographic Evaluation in Patients Receiving Anthracycline-Based Chemotherapy. American journal of cardiology. 116, 442-6.

Wang, X., Wang, X.L., Chen, H.L., Wu, D., Chen, J.X., Wang, X.X., Li, R.L., He, J.H., Mo, L., Cen, X., Wei, Y.Q., Jiang, W., 2014. Ghrelin inhibits doxorubicin cardiotoxicity by inhibiting excessive autophagy through AMPK and p38-MAPK. Biochemical pharmacology 88, 334-350.

Wang, Z., Wang, Y., Huang, Y., Lu, Q., Zheng, L., Hu, D., Feng, W., Liu, Y., Ji, K., Zhang, H., Fu, X., Li, X.,Chu, M.,Xiao,J., 2015b. bFGF regulates autophagy and ubiquitinated protein accumulation induced by myocardial ischemia/reperfusion via the activation of the PI3K/Akt/mTOR pathway. Scientific reports 19, 9287.

Xi, L., Zhu, S.G., Das, A., Chen, Q., Durrant, D., Hobbs, D.C., Lesnefsky, E.J., Kukreja, R.C., 2012. Dietary inorganic nitrate alleviates doxorubicin cardiotoxicity: mechanisms and implications. Nitric oxide 26, 274-284.

Zhou, Y.Y., Li, Y., Jiang, W.Q., Zhou, L.F., 2015. MAPK/JNK signalling: a potential autophagy regulation pathway. Bioscience reports 35 . 